\title{
Silicon Photonic Biochemical Sensor on Chip Based on Interferometry and Phase-Generated-Carrier Demodulation
}

\author{
Yisbel Eloisa Marin ${ }^{\circledR}$, Veronica Toccafondo, Philippe Velha, Simona Scarano ${ }^{\circledR}$, Stefano Tirelli, Alessandro Nottola, \\ Yoonah Jeong, Hyunpyo Jeon, Sanghun Kim, Maria Minunni ${ }^{\circledR}$, Fabrizio Di Pasquale, and Claudio J. Oton
}

\begin{abstract}
Integrated biochemical sensors based on MachZehnder interferometers (MZIs) rely on the evanescent field detection principle to monitor the phase shift induced by a refractive index change on the surface of the sensing arm, providing a high sensitivity thanks to the long interaction length with the analyte. This paper presents an integrated refractive index and biochemical sensor based on a pair of balanced MZIs realized on silicon-on-insulator. The device includes a thermal phase modulator in one of the branches of each MZI to extract univocally the induced phase change by applying active phase detection based on the phase generated carrier technique. The direct modulation of the MZI results in a simple configuration of the sensor, only requiring a fixed wavelength laser and a photodetector, enabling the possibility of full monolithic integration. The results of refractive index measurements show a detection limit down to $4.88 \times 10^{-7}$ RIU. Additionally, an immunoassay reports the binding of anti-bovine serum albumin (BSA) to BSA, with anti-BSA concentrations down to $33.33 \mathrm{nM}$.
\end{abstract}

Index Terms-Optical sensors, chemical and biological sensors, photonic integrated circuits, interferometry, phase modulation.

\section{INTRODUCTION}

I $\mathrm{N}$ THE past decades, there has been a growing interest in integrated photonic biochemical sensors for a wide range of applications, such as clinical diagnosis, food safety and chemical and biological warfare surveillance [1]. In particular, silicon

Manuscript received April 1, 2018; revised May 22, 2018; accepted June 8, 2018. Date of publication July 12, 2018; date of current version July 23, 2018. This work was supported by the Korean Institute of Science and Technology Europe, project number: 11896. (Corresponding author: Yisbel Eloisa Marin.)

Y. E. Marin, P. Velha, F. Di Pasquale, and C. J. Oton are with the Scuola Superiore Sant'Anna, TeCIP Institute, Pisa 56124, Italy (e-mail: y.marin@santannapisa.it; philippe.velha@santannapisa.it; f.dipasquale@ santannapisa.it; c.oton@santannapisa.it).

V. Toccafondo is with the Consorzio Nazionale Interuniversitario per le Telecomunicazioni, Pisa 56124, Italy (e-mail: veronica.toccafondo@cnit.it).

S. Scarano and M. Minunni are with the Dipartimento di Chimica "Ugo Schiff,” Universitá degli Studi di Firenze, Florence 50019, Italy (e-mail: simona. scarano@unifi.it; maria.minunni@unifi.it).

S. Tirelli and A. Nottola are with the Scuola Superiore Sant'Anna, TeCIP Institute, Pisa 56124, Italy, and also with the Consorzio Nazionale Interuniversitario per le Telecomunicazioni, Pisa 56124, Italy (e-mail: stefano.tirelli@cnit.it; anottola@cnit.it).

Y. Jeong, H. Jeon, and S. Kim are with the Korean Institute of Science and Technology - Europe, Saarbrücken 66123, Germany (e-mail: ya.jeong@kist-europe.de; hpjeon@kist-europe.de; shkim@kist-europe.de).

Color versions of one or more of the figures in this paper are available online at http://ieeexplore.ieee.org.

Digital Object Identifier 10.1109/JSTQE.2018.2854561 photonics is a technology platform with a complete toolbox of integrated components, like couplers, filters, modulators and detectors [2], which allows monolithic fabrication of optical systems such as integrated biochemical sensors. Although there are still challenges to be addressed in the integration of the light source, limiting the number of fully integrated devices currently available [3], [4], this platform allows to achieve high production volumes at a very low cost per chip, making it affordable to dispose the devices after one or a few measurements, enabling a variety of lab-on-chip (LOC) point-of-care applications.

Integrated-optic biochemical sensors are commonly based on the evanescent field detection principle, characterized by a high sensitivity and label-free detection capabilities [1]. This basic physical mechanism, allows one to detect changes in the effective refractive index $n_{\text {eff }}$ of a waveguide, enabling the device to act as a refractometer when the refractive index of the medium $n_{\ell}$ surrounding the waveguide changes, or as a biochemical sensor when a layer of adsorbed or bound molecules is formed on the surface of the waveguide [5]. Different photonic circuit configurations based on evanescent field detection have been proposed relying on different readout techniques to determine the refractive index change. Devices including ring resonators [6], [7], and photonic crystals [8], [9], provide very high sensitivities and small footprint, but rely on detection of changes in the transmission spectrum, requiring expensive and bulky spectrum analyzers for signal processing, whereas devices based on Mach Zehnder interferometers (MZI) rely on measuring the phase change induced by a refractive index variation in one of the two arms [10]. MZIs are particularly attractive thanks to the high sensitivity provided by their long interaction length with the analyte; however, there is a trade-off between the size of the device and the sensitivity, since the latter is proportional to the sensing arm length. Including photonic crystals in one of the arms of the MZI can provide a fivefold increase in the sensitivity and 400 times reduction in footprint of a common MZI [11], but there is still the limitation of relying on spectrometry for accurate interrogation. Conventional MZIs can be interrogated using simpler interrogation schemes. The most straight forward technique would be through direct output intensity measurement; however, there are potential issues related to power or wavelength fluctuations of the source as well as to the ambiguity in the phase estimation and sensitivity fading close to the maxima and minima, due to the sinusoidal response of the MZI. A 


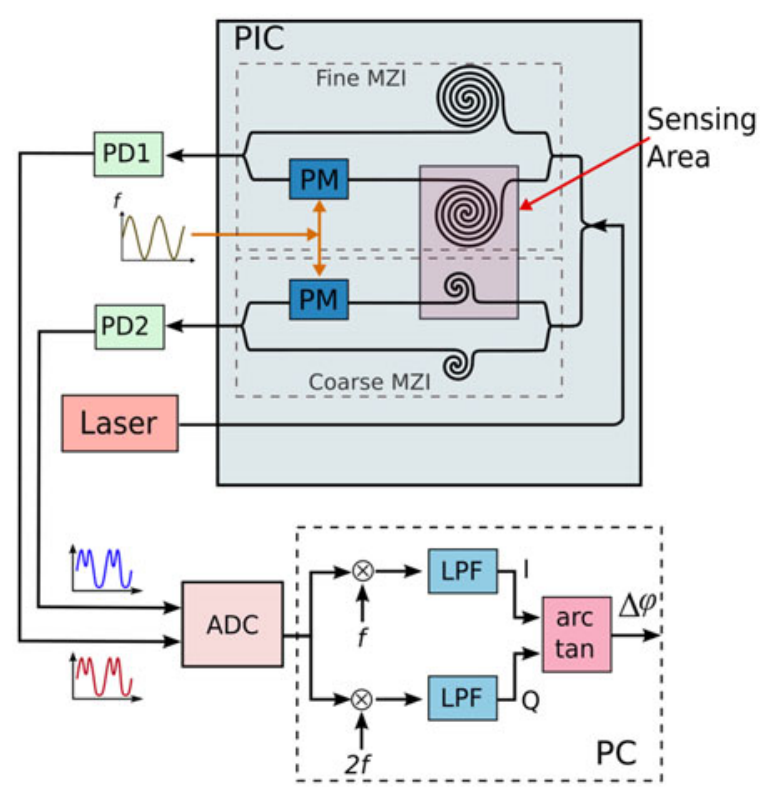

Fig. 1. Schematic for active phase demodulation using the phase generated carrier (PGC) technique for biochemical sensing applications. PIC: Photonic Integrated Circuit; PM: Phase Modulator; PD: Photodetector; ADC: Analog-toDigital Converter; LPF: Low-Pass Filter; PC: Personal Computer.

possible interrogation method that solves these issues consists of applying a pseudoheterodyne phase modulation in one of the arms of the MZI [12], tracking then the phase variations at the output with respect to a reference trigger. This technique can provide an acceptable performance but requires the use of expensive equipment like a lock-in amplifier, and it is also affected by a discontinuity in the applied modulation profile. A different method that also effectively addresses these issues consists of applying phase modulation to the source [13], which does not require electrical probes. However, the specific optical source control requirements could complicate even more the integration of all the components in order to obtain a LOC device; moreover, the unbalanced characteristic of the MZI would make the device sensitive to thermal fluctuations and wavelength variations of the source.

In this paper we present an integrated photonic circuit for refractive index measurement and biochemical sensing consisting of a balanced Mach-Zehnder interferometer and an active phase detection technique based on phase generated carrier (PGC) demodulation. This method allows univocal determination of the phase variations without sensitivity fading, requiring only a fixed laser and a photodetector, which could potentially be integrated on chip [2], resulting in a low-cost, robust interrogation system for point-of-care applications. This paper is a more in-depth work with respect to the recent conference publication [14].

\section{CONCEPT}

The phase generated carrier (PGC) technique was first proposed for fiber-optic based interferometers [15] and consists of applying an external sinusoidal phase modulation on one of the branches of an MZI, as shown in Fig. 1, with a frequency $f$ much higher than the response frequency of the sensing interactions, and amplitude $C$, yielding the following signal at the output of the MZI:

$$
I=A+B \cos [C \cos (2 \pi f t)+\Delta \varphi(t)],
$$

where $A$ is the DC component, $B$ depends on the mixing efficiency of the MZI and $\Delta \varphi(\mathrm{t})$ is the phase change induced by a refractive index variation in the area surrounding the sensing arm of the MZI. Expanding this expression in terms of Bessel functions, results in:

$$
\begin{aligned}
I= & A+B\left\{\left[J_{0}(C)+2 \sum_{k=1}^{\infty}(-1)^{k} J_{2 k}(C) \cos 2 k \omega_{0} t\right]\right. \\
& \times \cos \Delta \varphi(t) \\
& \left.-\left[2 \sum_{k=0}^{\infty}(-1)^{k} J_{2 k+1}(C) \cos (2 k+1) \omega_{0} t\right] \times \sin \Delta \varphi(t)\right\} .
\end{aligned}
$$

Mixing (2) at odd $(f)$ and even (2f) multiples of the modulating frequency $f$ and applying a low-pass filter to remove the spectral components above the band of interest results in the signals $S_{1}=J_{1}(C) \sin \Delta \varphi(t)$ and $S_{2}=J_{2}(C) \cos \Delta \varphi(t)$, where $J_{1}(C)$ and $J_{2}(C)$ are the Bessel functions of first and second order respectively, evaluated in $C$. By choosing the amplitude of the modulation such that $C=0.84 \pi$, the condition $J_{1}(C)=$ $J_{2}(C)$ is satisfied, making it possible to univocally extract $\Delta \varphi(\mathrm{t})$ through the arctangent method as:

$$
\Delta \varphi(t)=\arctan \left(\frac{S_{1}}{S_{2}}\right)
$$

solving the sensitivity fading and source intensity fluctuation issues of the standard MZI. Finally, a refractive index change $\Delta n_{\ell}$ of a liquid covering the sensing window in one of the branches of the MZI can be determined from the measured phase change $\Delta \varphi(\mathrm{t})$ through the relation:

$$
\Delta \varphi=\frac{2 \pi}{\lambda} L \Delta n_{e f f}=\frac{2 \pi}{\lambda} L \frac{\partial n_{e f f}}{\partial n_{\ell}} \Delta n_{\ell},
$$

where $L$ is the interaction length or length of the MZI arm exposed to the liquid, $\lambda$ is the wavelength of the optical source and $\Delta n_{e f f}$ is the change in effective refractive index of the sensing arm of the MZI.

Considering an initially balanced MZI, removing the cladding from one of the branches would result in a very unbalanced device, due to the difference in the refractive index between the claddings (silica and the liquid analyte). The free spectral range (FSR) of this device is given by:

$$
\lambda_{F S R}=\frac{\lambda^{2}}{L_{\text {sens }} \times n_{g, \text { sens }}-L_{\text {ref }} \times n_{g, r e f}},
$$

where $L_{\text {sens }}$ and $L_{\text {ref }}$ are the lengths of the sensing and reference arms, and $n_{g, \text { sens }}$ and $n_{g, r e f}$ are the group indices of the waveguides of the sensing and reference arms respectively. The cladding difference between the arms would yield a small FSR, making the device susceptible to temperature and source 


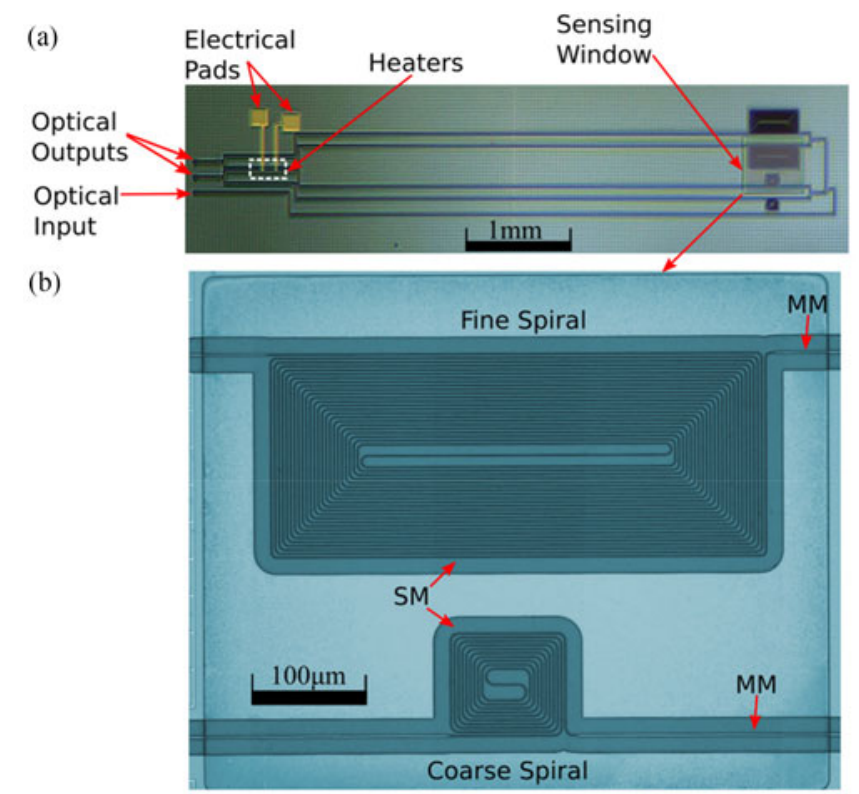

Fig. 2. (a) Optical photograph of the device. Total size: $5.8 \mathrm{~mm} \times 900 \mu \mathrm{m}$. (b) Optical photograph of the sensing area. The upper spiral corresponds to the fine circuit, whereas the lower spiral corresponds to the coarse circuit. SM: Single-mode; MM: Multi-mode.

wavelength fluctuations. One solution to this issue is to satisfy the condition:

$$
L_{\text {sens }} \times n_{g, \text { sens }}=L_{\text {ref }} \times n_{g, r e f},
$$

by properly selecting the arm lengths, resulting in an MZI with ideally no fringes in the spectrum, however fabrication errors could cause the appearance of a few fringes.

\section{DESIGN AND FABRICATION OF THE DEVICE}

The optical design consisted of two balanced MZIs, one with a shorter interaction length, so-called coarse, and another with a longer interaction length, so-called fine, as shown in Fig. 2(a), which can be monitored simultaneously in order to maximize the measuring range and sensitivity. The interaction length of each MZI was wound in spirals to reduce the size of the sensing area, as shown in Fig. 2(b). The cladding over these spirals was removed to expose the waveguide to the analyte, while on the other arm an almost identical spiral remained covered and acted as a reference, so that a change in the refractive index of the analyte would induce a phase difference at the output of the MZIs. The lengths of the exposed spirals for the coarse and fine interferometers were $2.5 \mathrm{~mm}$ and $22 \mathrm{~mm}$ respectively. The length difference between the reference and sensing spirals in the two MZIs was not identical to satisfy the condition in (6); the reference branch of the coarse interferometer was $1.57 \%$ longer in order to compensate for the group index difference thus reducing the fringe density (see Fig. 3(a)) and therefore the sensitivity to wavelength and temperature fluctuations. For the fine interferometer the group index matching condition in (6) was not met, with a reference spiral unintentionally longer by $8.17 \%$, which caused a higher fringe density, as shown in Fig. 3(b), and therefore higher instability to

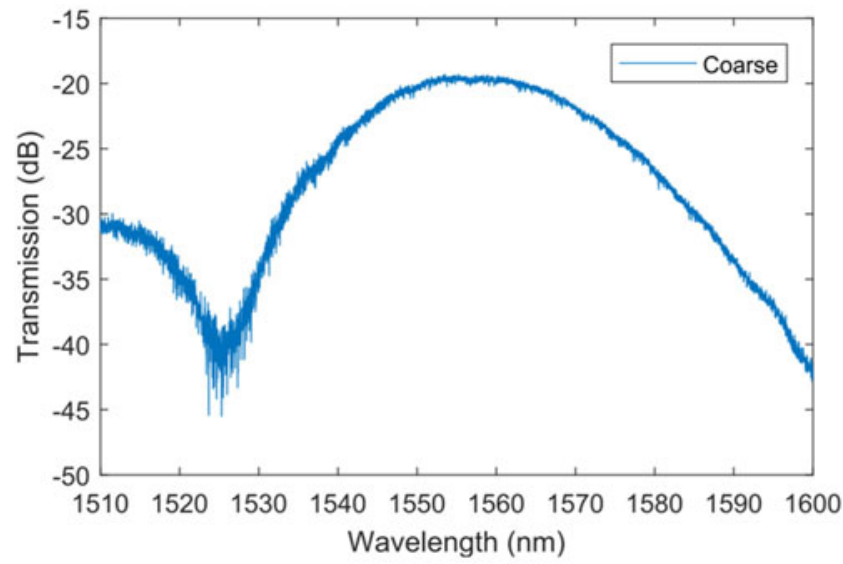

(a)

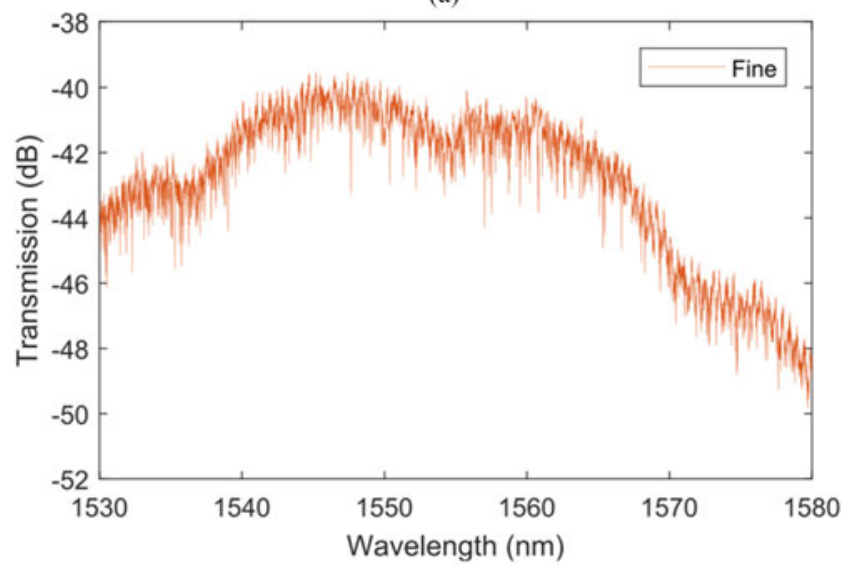

(b)

Fig. 3. (a) Spectrum of the coarse MZI when the sensing spiral was exposed to deionized water. (b) Spectrum of the fine MZI when the sensing spiral was exposed to deionized water.

wavelength and thermal fluctuations. It should be noted that the lower visibility for the fine circuit is due to the higher total propagation loss $\left(\alpha_{\text {total }}\right)$ of the longer spirals, given by $\alpha_{\text {total }}=\alpha_{\mathrm{wg}} \times L$, where $\alpha_{\mathrm{wg}}$ is the waveguide losses in $\mathrm{dB} / \mathrm{cm}$ (mainly attributed to sidewall roughness), and $L$ is the length of the arm in $\mathrm{cm}$. Although the spectrum for the fine MZI in Fig. 3 shows a lower fringe contrast and higher noise than for the coarse, both attributed to the higher spiral length, it was possible to use the two devices to measure simultaneously.

The photonic integrated circuit, with a total size of $5.8 \mathrm{~mm}$ $\times 900 \mu \mathrm{m}$, as shown in Fig. 2(a), was fabricated in-house at the Integrated Photonic Technologies Center (INPHOTEC). The structures were patterned on a silicon-on-insulator wafer, with a 220-nm-thick silicon layer and 3- $\mu \mathrm{m}$-thick buried oxide, and later covered by a $1.5-\mu \mathrm{m}$-thick layer of $\mathrm{SiO}_{2}$ cladding layer. A sensing region of $\sim 500 \mu \mathrm{m} \times 500 \mu \mathrm{m}$ was defined by masking a part of the device and successively wet-etching the $\mathrm{SiO}_{2}$ cladding layer, in order to expose one spiral from each MZI, as shown in Fig. 2(a). $480 \mathrm{~nm}$-wide single-mode waveguides were used for spirals, bends and waveguides at the inputs and outputs of the multimode interferometers (MMIs) that split and combine the signals propagating through the interferometer. The remaining straight waveguides had a width of $1.5 \mu \mathrm{m}$ with parabolic 


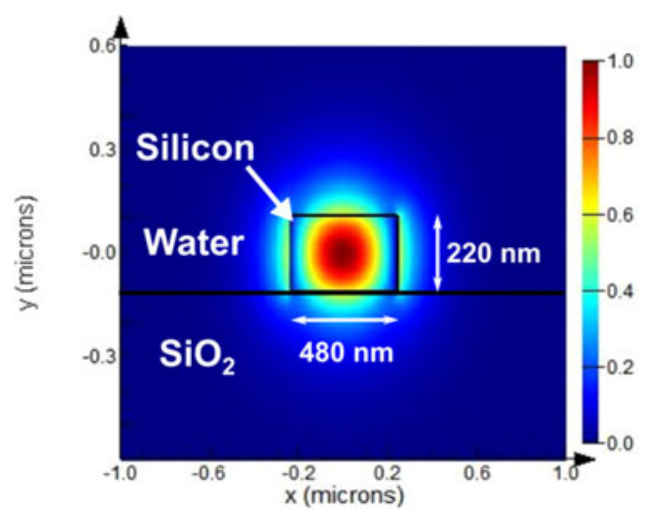

Fig. 4. Electric field $\left(E_{X}\right)$ profile of the propagating fundamental TE-mode of a $220 \mathrm{~nm} \times 480 \mathrm{~nm}$ waveguide. The evanescent field at the top and both sides interact with the liquid.

tapers at the ends to guarantee a single-mode propagation. The light was coupled in and out of the device using TE-optimized focusing grating couplers with a coupling loss of $\sim 6 \mathrm{~dB}$ (measured from a reference sample). Finally, the internal branches of both MZIs included a thermal phase modulator, which consisted of a metal Ti heater track which was $100 \mu \mathrm{m}$ long and $5.25 \mu \mathrm{m}$ wide, with $\mathrm{Ti} / \mathrm{Au}$ paths for the electric probing. This modulator had a 1/e time-constant of $\sim 7 \mu$ s and $\mathrm{a}_{2 \pi}$ of $3.55 \mathrm{~V}$. The heaters and paths were placed far from the sensing window in order to avoid contact between the electric probes and the liquids under test.

Fig. 4 shows the electric field $\left(\mathrm{E}_{\mathrm{X}}\right)$ profile of the propagating fundamental TE-mode of the sensing waveguides obtained using a finite difference eigenmode (FDE) solver (MODE Solutions from Lumerical Inc.). The refractive index model for the silicon waveguides was obtained from [16]. For the lower cladding the refractive index was set to 1.444 , corresponding to $\mathrm{SiO}_{2}$, whereas the refractive index of the top cladding was set to 1.32 , corresponding to deionize water (DIW) at $1550 \mathrm{~nm}$. Regarding the simulation area of the FDE, this was defined normal to the propagation direction, with a width of $2.08 \mu \mathrm{m}$ and thickness of $1.22 \mu \mathrm{m}$. The mesh of the simulation area had maximum step of $0.01 \mu \mathrm{m}$ in $d x$ and $d y$, and minimum step of $1 \times 10^{-6} \mu \mathrm{m}$. Finally, the boundary conditions were set to perfectly matched layer (PML) in all directions. The results of the simulation confirm that the evanescent field interacts with the liquid (DIW) at the top and both sides of the waveguide. The device characterization and sensing tests were performed by placing the chip on a micropositioner station with a TEC fixed at $20^{\circ} \mathrm{C}$. A polydimethylsiloxane (PDMS) cell with two outlets was fixed on top of the sensing area using a piece of plexiglass and screws to avoid liquid leakage. Teflon tubes were used to connect the PDMS cell to a multi-port switch valve and to a pump that pulled the liquid with a constant flux. The multi-port switch was at the same time connected to Eppendorf containers that stored the different liquids under test.

\section{EXPERIMENTS AND RESULTS}

The experimental setup scheme is shown in Fig. 1. A tunable external cavity laser was used as a light source, working at a wavelength of $1560 \mathrm{~nm}$, and with an optical power output of
$10 \mathrm{~mW}$. The input and outputs of the device were aligned on the same side of the chip (see Fig. 2(a)), allowing to use a fiber array to couple the light in and out of the device. At the output, two InGaAs based photoreceivers with a trans-impedance amplifier (one per MZI) were used to detect the phase modulated signals. The bandwidth of the photoreceivers, limited by the amplifier settings, was $320 \mathrm{kHz}$ for the coarse port and $90 \mathrm{kHz}$ for the fine port; note that the lower bandwidth for the fine port is due to the higher amplifier gain required to compensate for the additional propagation losses. Finally, the electrical signals from the photodetectors were coupled to an analog-to-digital converter (ADC) operating at $100 \mathrm{kSamples} / \mathrm{s}$ for data acquisition and the processing, including mixing, low-pass filtering, and unwrapping of the arctangent signal, was performed in real time via software on a standard PC. The PGC modulation frequency was set to $6.4 \mathrm{kHz}$ and the applied low-pass filter had a cut-off frequency of $1 \mathrm{kHz}$.

\section{A. Refractometry Experiment}

The refractive index measurements were performed using glycerol in deionized water (DIW) dilutions, with concentrations in volume of $1 \%, 1.1 \%, 2 \%$ and $5 \%$. Initially, the refractive index of DIW was measured starting at $t=0 \mathrm{~s}$ to establish a baseline, and then, the valve was switched to the first concentration of glycerol, alternating later with DIW between the different concentrations in intervals of $\sim 600 \mathrm{~s}$. Fig. 5(a) and (b) show that after introducing the diluted glycerol a fast phase variation occurred, which stabilized after keeping a continuous flux of the same concentration, resulting in a response time (time required for reaching $95 \%$ of the final response) of $\sim 111.15 \mathrm{~s}$ for the fine and $\sim 108.5 \mathrm{~s}$ for the coarse, and then returned to the baseline level when switching back to DIW. The phase change measured with the fine MZI (see Fig. 5(b)) was, as expected, higher than the measured using the coarse MZI (see Fig. 5(a)). There is a $\sim 8.2$ factor difference between the two measurements, not fully consistent with the length ratio of the spirals in the coarse and fine MZIs which is 8.8; the reason for this small discrepancy is due to the short straight waveguide segment which was exposed in the coarse interferometer, which had a width of $1.5 \mu \mathrm{m}$.

In order to study the linearity of the MZIs, we plotted the phase variation normalized to the maximum phase shift of each trace as shown in Fig. 6(a). Fig. 6(b) reports a plot representing phase variation versus concentration for several repetitions showing a good linear dependence (in dashed lines).

The refractive indices for DIW and a glycerol aqueous solution with $2 \%$ volume concentration at $25^{\circ} \mathrm{C}$ are $n(0 \%)=1.3165$ and $n(2 \%)=1.3193$ respectively [17]. From these values it is possible to estimate the effective index change of the sensing waveguide when the liquid surrounding it changes from water to a $2 \%$ dilution of glycerol in DIW, calculated using a finite difference eigenmode solver (MODE Solutions from Lumerical). The parameters of the simulation were the same as in Section III, but in this case, we changed the refractive index of the upper cladding to the one corresponding to a $2 \%$ glycerol aqueous solution. The results showed that a variation in the refractive index of the liquid causes a change in the effective index of the waveguide $\left(\partial n_{e f f} / \partial n_{\ell}\right)$ of $16.46 \%$ for the $480 \mathrm{~nm}$ - 


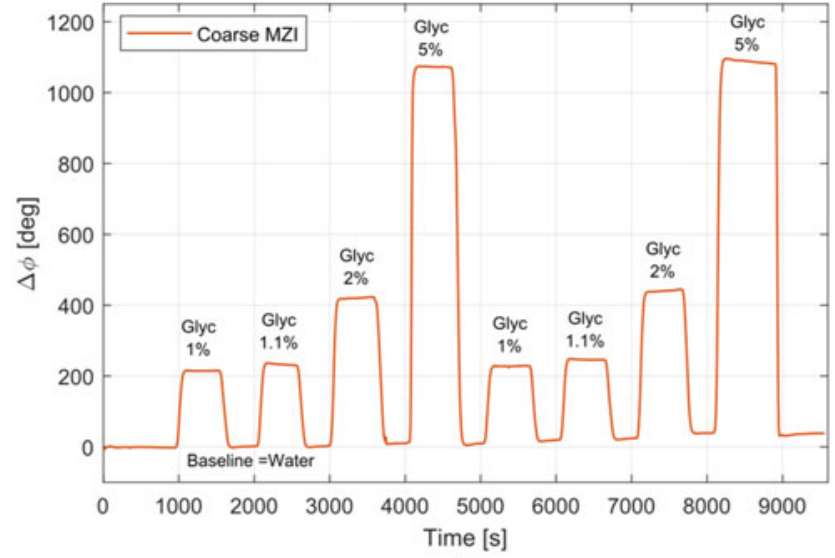

(a)

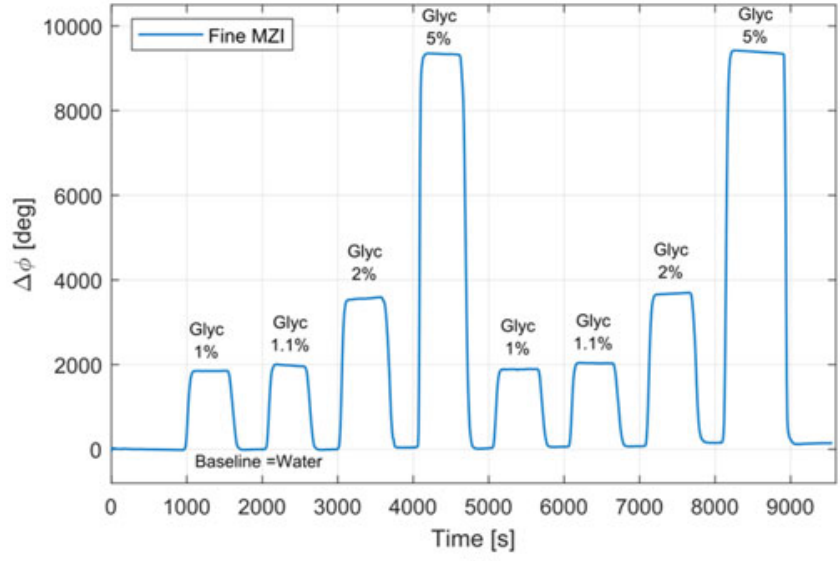

(b)

Fig. 5. Phase shift versus time for different glycerol concentrations in water measured using (a) the coarse MZI and (b) the fine MZI.

wide spiral and $4.56 \%$ for the $1.5 \mu \mathrm{m}$-wide straight waveguide inside the sensing window. From these values, we estimated the expected phase variations for a change in concentration of glycerol from $0 \%$ to $2 \%$ of $279.69^{\circ}$ for the coarse MZI, and $2358.56^{\circ}$ for the fine MZI. The actual phase variation measured with the device was $431.8^{\circ}$ for the coarse MZI and $3549.675^{\circ}$ for the fine MZI, which are higher than estimated. These experimental values result in a $\partial n_{e f f} / \partial n_{\ell}$ coefficient of $25.82 \%$ for the $480 \mathrm{~nm}$-wide waveguide, which is higher than the estimated value. We attribute this discrepancy to the depth of the upper-cladding etching, which in the simulation was considered to be precisely aligned to the bottom of the waveguide, while in the real case there might have been some cladding over-etching combined with a partial etching of the waveguide core, leading to a higher sensitivity.

The performance of the device was evaluated in terms of sensitivity, detection limit and temperature stability. The sensitivity of the device $S=\Delta \phi / \Delta n_{\ell}$ represents the phase shift per refractive index unit (RIU) of the liquid; for the coarse MZI we obtained a sensitivity of $S=1.565 \times 10^{5} \mathrm{deg} / \mathrm{RIU}$, equivalent to $S=2.731 \times 10^{3} \mathrm{rad} / \mathrm{RIU}$, and for the fine MZI we obtained $S=$ $1.315 \times 10^{6} \mathrm{deg} / \mathrm{RIU}$, equivalent to $S=2.295 \times 10^{4} \mathrm{rad} / \mathrm{RIU}$. Regarding the detection limit, which is the minimum refractive index variation that can be measured by the device and can be expressed as $D L=3 \sigma / S$, where $\sigma$ is the noise level, we found

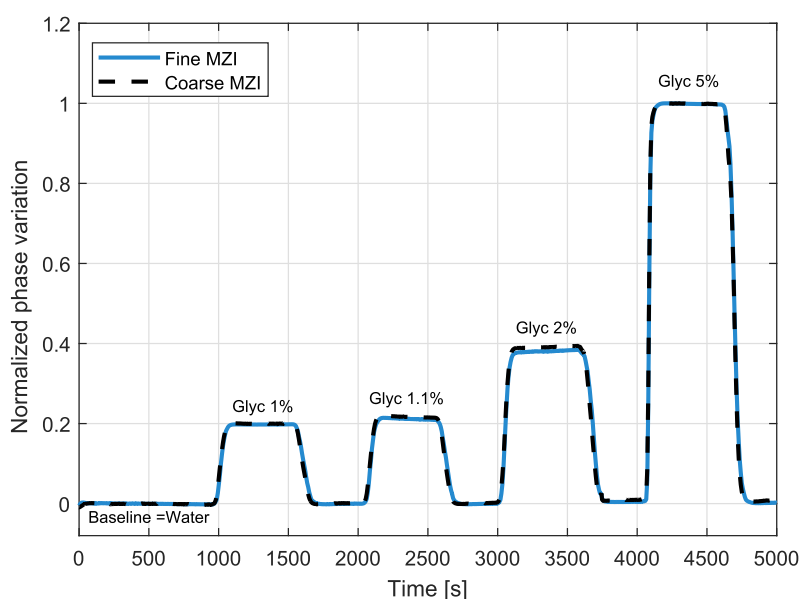

(a)

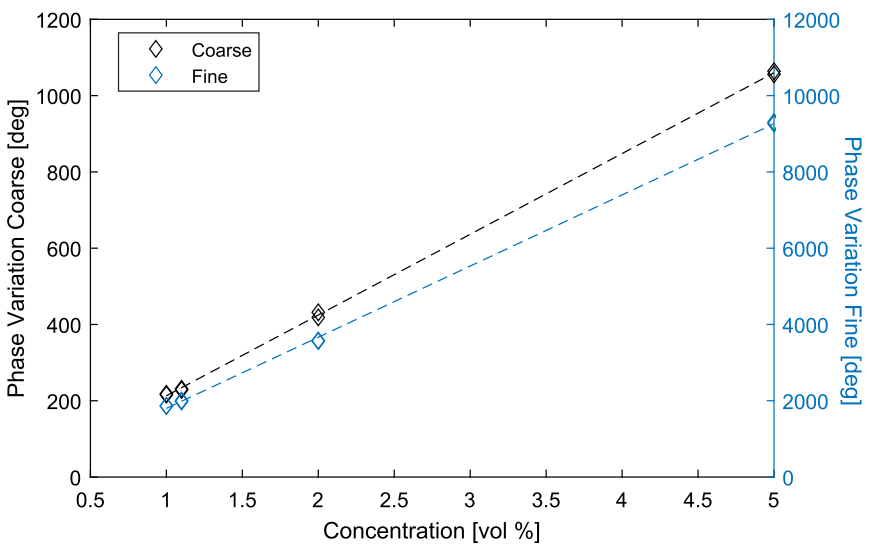

(b)

Fig. 6. Phase shift versus time for different glycerol concentrations in water measured using the (a) coarse MZI and (b) fine MZI.

values of $3 \sigma=0.276 \mathrm{deg}$ (see Fig. $7\left(\right.$ a)), and $D L=1.763 \times 10^{-6}$ RIU for the coarse MZI, and $3 \sigma=0.6417 \mathrm{deg}$ (see Fig. 7(b)) and $D L=4.882 \times 10^{-7} \mathrm{RIU}$ for the fine. It should be noted that the noise was calculated over an interval that includes thermal drift, and as seen in Fig. 7, both traces present proportional variations that might be due to real refractive index variations in the analyte or other factors that need to be studied in more detail in future work, so the noise is not strictly Gaussian and it is probably overestimated. Finally, we found that the phase variation with respect to a change of temperature on the chip was $12.72 \mathrm{deg} /{ }^{\circ} \mathrm{C}$ for the coarse MZI and $163.79 \mathrm{deg} /{ }^{\circ} \mathrm{C}$ for the fine MZI, which is due to the coarse circuit being better balanced, as evidenced in Fig. 3(a) and (b). Even though these values of thermal sensitivity allowed us to acquire clean phase profiles, the device performance can be improved by designing an athermal interferometer able to cancel out the temperature-dependent phase shift induced by the different thermo-optic coefficients of the three materials involved: silicon, silica and water. The athermal interferometer design is however beyond the scope of this work and will be the subject of a future work.

\section{B. Biosensing Experiment}

1) Sample Functionalization and Biomolecule Attachment: The photonic devices were modified to achieve the covalent im- 


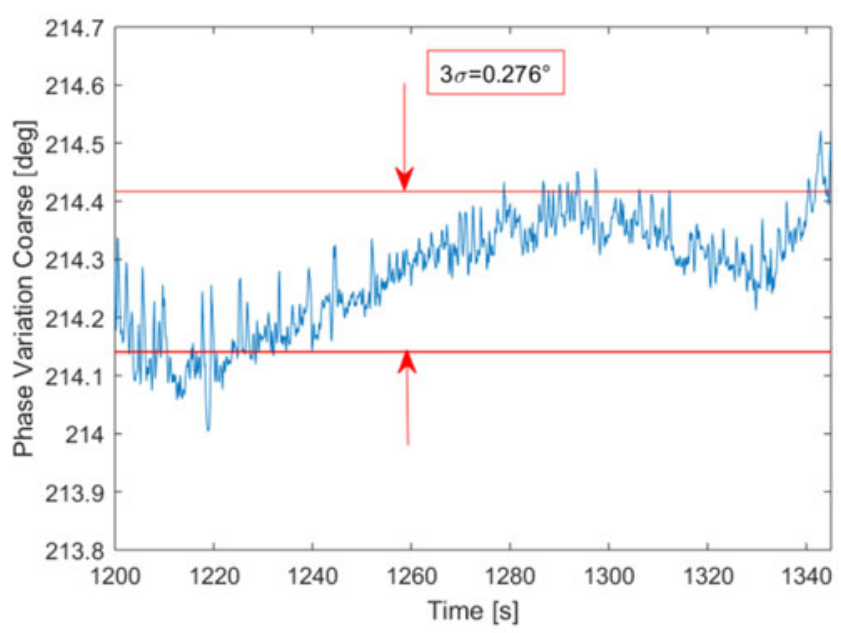

(a)

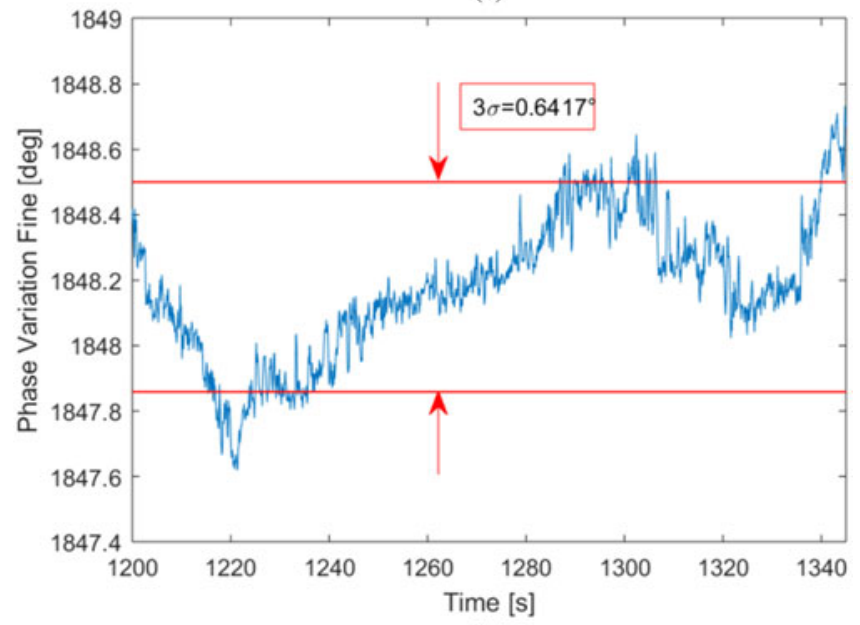

(b)

Fig. 7. Zoom of the phase variation response showing the rms noise measured for $1 \%$ glycerol using the (a) coarse MZI and (b) fine MZI.

mobilization of a biological recognition element at their surface. The immobilization protocol consisted basically in the linking of a standard reference protein, i.e., Bovine Serum Albumin (BSA), on one of the two sensing windows present on a chip with two replicas of the device. The other one was treated as a control surface and therefore it underwent all the modification treatment, excluding the final BSA addition. This assured the real-time subtraction of possible unspecific signal due to the interaction of the analyte, anti-BSA antibody (polyclonal anti-BSA, Sigma-Aldrich), with the transducer surface. Due to the strong hydrophobic nature of the silicon wafer, the first step involved the surface oxidation by piranha solution $(3: 1$ $\mathrm{H} 2 \mathrm{SO} 4: 30 \% \mathrm{H} 2 \mathrm{O} 2,10 \mathrm{~min}$ ). Afterward, the devices were thoroughly washed with MilliQ water (ultrapure water at $18.2 \mathrm{M} \Omega \times$ $\mathrm{cm}$ at $25^{\circ} \mathrm{C}$ ). Finally, the chips were dried under a nitrogen stream and promptly used for further modification to avoid the loss of hydrophilicity achieved during the oxidation process. The chips were then organically modified using standard silanization chemistry. The entire chip was exposed to a $5 \%$ solution of 3-aminopropyltriethoxysilane (APTES) in 95\% ethanol for $60 \mathrm{~min}$, followed by the removal of residual siloxane by rinsing in $95 \%$ ethanol.

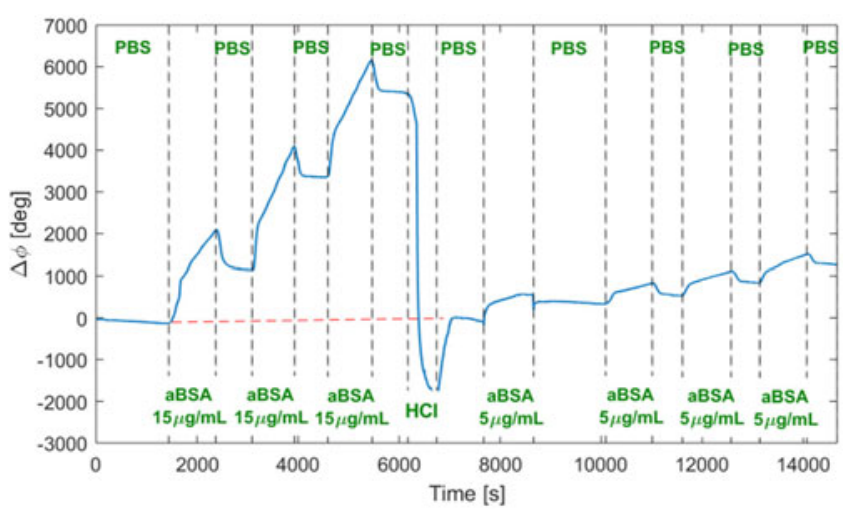

Fig. 8. Phase shift versus time caused by the antigen/antibody a-BSA/BSA immunoreaction at $5 \mu \mathrm{g} / \mathrm{mL}$ and $15 \mu \mathrm{g} / \mathrm{mL}$ concentrations.

The covalent immobilization of Bovine Serum Albumin (BSA) to the sensor surface was achieved in two steps using amino-coupling reaction. The freshly silanized surfaces were exposed to a $2.5 \%$ solution of glutaraldehyde in phosphate buffered solution (PBS) for $1 \mathrm{~h}$. After washing with PBS buffer (0.1 M phosphate buffered solution, $\mathrm{pH}$ 7.4) and MilliQ water, the reactive aldehyde moiety exposed was conjugated to the BSA (200 mg/L). After reacting for $1 \mathrm{~h}$ at room temperature to maximize the amount of covalently immobilized antibody, a PBS buffer rinse was then carried out to remove noncovalently bound protein. After drying, the photonic devices were tested in biosensing experiments by assaying their refractive index susceptibility upon the specific binding of the target analyte on the receptor moiety. As negative control, a nonspecific antibody, anti-goat IgG was also tested.

2) Biochemical Sensing Results: For the demonstration of the biosensing capabilities of the device we immobilized Bovine Serum Albumin (BSA) protein on the fine MZI, and observed the specific interaction with the relative anti-BSA antibody. Through this approach, the monitoring of the immunoreaction can be performed in real time and without the use of any label. The fine MZI was selected as substrate for its higher sensitivity. As a proof of concept, the immunosensor was assayed with two different concentrations of anti-BSA, $5 \mu \mathrm{g} / \mathrm{mL}(33.3 \mathrm{nM})$ and $15 \mu \mathrm{g} / \mathrm{mL}(99.9 \mathrm{nM})$ respectively, in a buffer solution $(0.1 \mathrm{M}$ phosphate buffered solution, $\mathrm{pH}$ 7.4). The flow rate was fixed at $10 \mu \mathrm{L} / \mathrm{min}$ and the running buffer was the same PBS solution used for anti-BSA dilutions. The immunosensor behavior is shown in Fig. 8. At $t=0 \mathrm{~s}$ the buffer flows, providing a stable base line, followed at $t=1440 \mathrm{~s}$ by a $15 \mu \mathrm{g} / \mathrm{mL}$ anti-BSA injection (for $\sim 15 \mathrm{~min}$ ) producing at first a fast change in the phase, due to the refractive index difference between the buffer and the anti-BSA solution. The binding of anti-BSA on the BSA antigen is displayed and recognized as a constant increase in the sensor signal up to the washing phase, where a decrease in the signal can be observed, corresponding to the washing off of the anti-BSA excess. Then, a second and third injection of $15 \mu \mathrm{g} / \mathrm{mL}$ anti-BSA was applied, with washing steps in between, followed by the regenerating solution $(\mathrm{HCl}, 10 \mathrm{mM}$ for $\sim 2 \mathrm{~min}$ ) to dissociate the surface immunocomplex (BSA/aBSA), and finally by PBS to recover the system baseline. As evidenced in Fig. 8, the photonic immunosensor can be used for repeated measurement cycles 


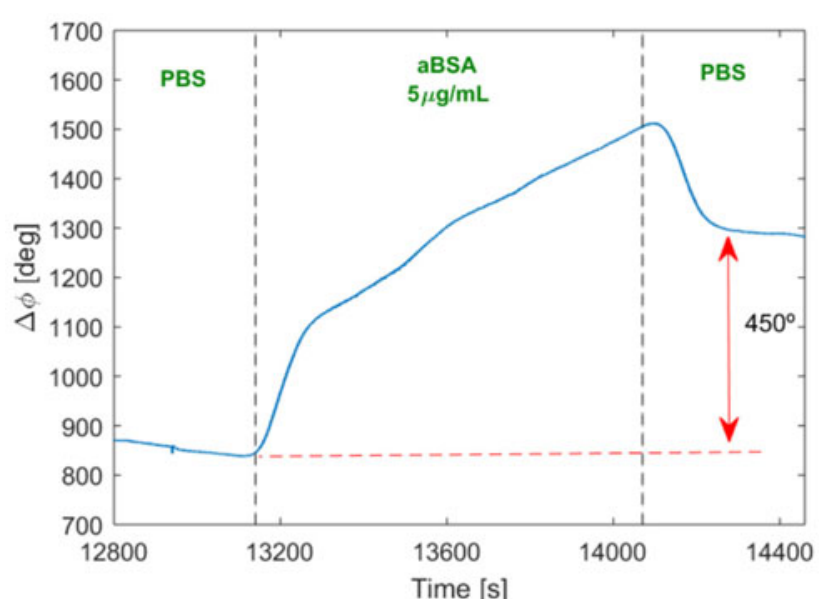

(a)

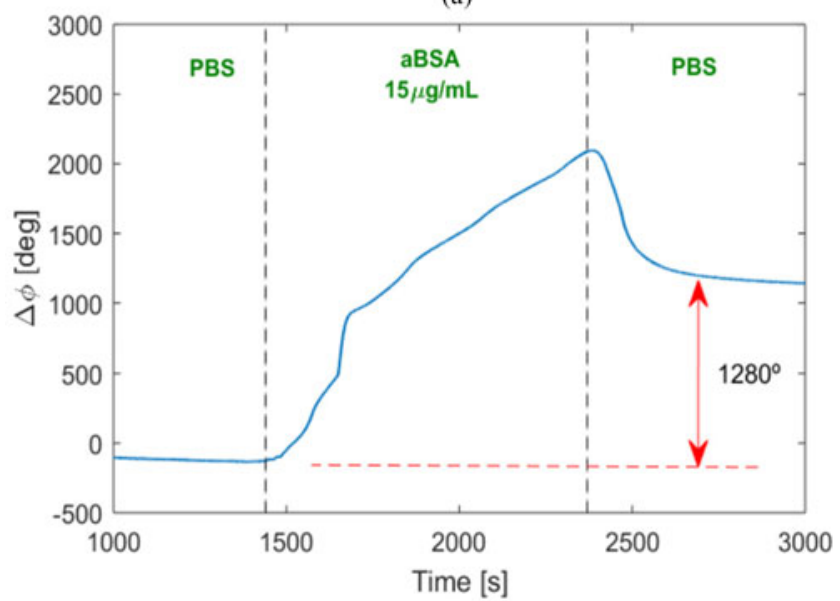

(b)

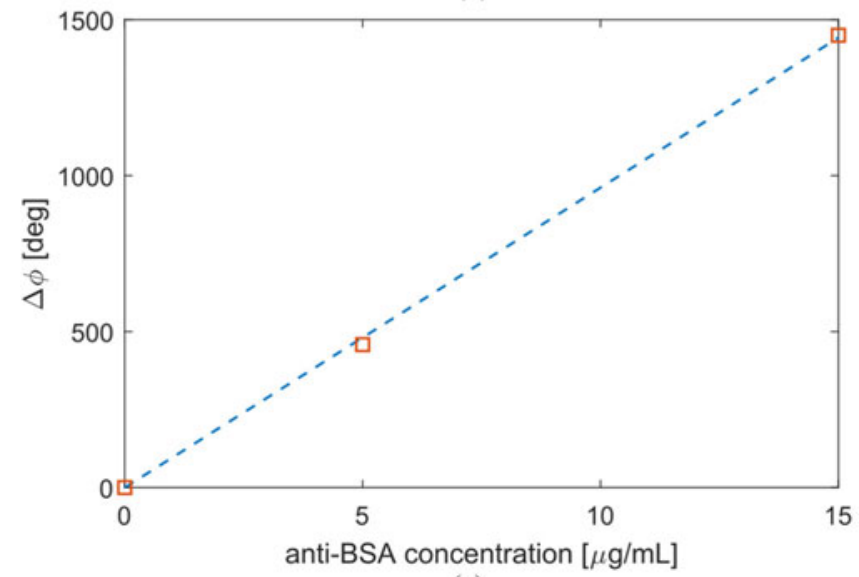

(c)

Fig. 9. Zoom of the phase shift versus time caused by the antigen/antibody anti-BSA/BSA immunoreaction at (a) $5 \mu \mathrm{g} / \mathrm{mL}$ and (b) $15 \mu \mathrm{g} / \mathrm{mL}$ concentrations. (c) Dose response plot showing the linear correlation obtained with anti-BSA up to $15 \mu \mathrm{g} / \mathrm{mL}$.

since, after the $\mathrm{HCl}$ solution exposure and buffer surface washing, the baseline successfully returned to the initial one recorded at $t=0 \mathrm{~s}$, i.e., before the first anti-BSA solution injection.

Further measurements were performed with a lower antiBSA concentration $(5 \mu \mathrm{g} / \mathrm{mL})$, to demonstrate the ability of the biosensor to provide a quantitative signal and discern among the different anti-BSA concentrations. As in the previous mea-

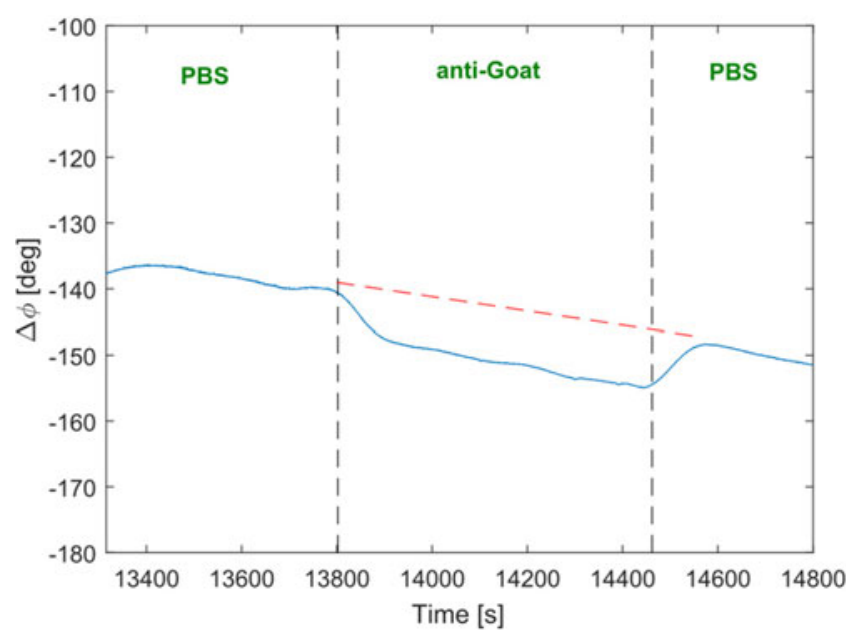

Fig. 10. Phase shift versus time caused when applying the anti-Goat at $10 \mu \mathrm{g} / \mathrm{mL}$ on the BSA activated surface.

surements, $5 \mu \mathrm{g} / \mathrm{mL}$ anti-BSA was injected in series with a washing step in between to remove the unbound antibody. As expected, the phase change (refractive index change) shown in Fig. 8 (zoomed in Fig. 9(a)), was smaller and proportional to the concentration tested. By plotting the obtained phase shift versus concentration, a linear correlation was obtained as reported in Fig. 9(c). The final step of our demonstration was to apply a control anti-body (anti-Goat solution in PBS at $10 \mu \mathrm{g} / \mathrm{mL}$ ) to the device with immobilized BSA on the surface to determine if the immunoreaction was indeed selective. The test was performed after applying the regenerating solution following the binding test of anti-BSA on the BSA. Initially a flow of the buffer solution was applied for $10 \mathrm{~min}$, to establish a stable baseline, followed by an anti-Goat injection for $10 \mathrm{~min}$, and finally by the washing off phase with PBS, in order to remove any antiGoat excess. Fig. 10 shows the phase change curve, where it can be observed that there is a small negative phase shift when the anti-Goat solution gets in contact with the sensing area, but after applying PBS again the baseline returns to the initial value, indicating that no binding took place, hence demonstrating the selectivity of the immunosensor. The negative sign of the phase change is likely due to a difference in the buffer solutions used for the baseline and for preparing the anti-Goat dilution.

\section{CONCLUSION}

In this paper we presented a refractive index and biochemical sensor based on integrated silicon photonics. The circuit, consisting of a Mach Zehnder interferometer, exploits an active demodulation technique based on phase generated carrier (PGC) and the evanescent detection mechanism to measure refractive index changes in the surface of the exposed waveguides. We demonstrated a refractive index detection limit of $4.882 \times$ $10^{-7}$ RIU, and specific detection of BSA protein at concentrations down to $5 \mu \mathrm{g} / \mathrm{mL}(33.3 \mathrm{nM})$. The possibility of integration of the source and detectors on-chip indicates its potential of becoming a low-cost solution for a wide variety of sensing applications. 


\section{REFERENCES}

[1] M. Estevez, M. Alvarez, and L. Lechuga, "Integrated optical devices for lab-on-a-chip biosensing applications," Laser Photon. Rev., vol. 6, pp. 463-487, 2012.

[2] A. Samusenko et al., "Integrated silicon photodetector for lab-on-chip sensor platforms," Proc. SPIE, vol. 9520, 2015, Art. no. 95200D.

[3] K. Misiakos et al., "Fully integrated monolithic optoelectronic transducer for real-time protein and DNA detection: The NEMOSLAB approach," Biosens. Bioelectron., vol. 26, no. 4, pp. 1528-1535, 2010.

[4] K. Misiakos et al., "All-silicon monolithic Mach-Zehnder interferometer as a refractive index and bio-chemical sensor," Opt. Express, vol. 22, pp. 26803-26813, 2014.

[5] W. Lukosz, "Integrated optical chemical and direct biochemical sensors,' Sens. Actuators B, Chem., vol. 29, no. 1, pp. 37-50, 1995.

[6] M. Iqbal et al., "Label-free biosensor arrays based on silicon ring resonators and high-speed optical scanning instrumentation," IEEE J. Sel. Topics Quantum Electron., vol. 16, no. 3, pp. 654-661, May/Jun. 2010.

[7] S. Mudumba et al., "Photonic ring resonance is a versatile platform for performing multiplex immunoassays in real time," J. Immunol. Method, vol. 448, pp. 34-43, 2017.

[8] S. Hu et al., "Enhancing the sensitivity of label-free silicon photonic biosensors through increased probe molecule density," ACS Photon., vol. 1, no. 7, pp. 590-597, 2014.

[9] S. M. Lo et al., "Photonic crystal microring resonator for label-free biosensing," Opt. Express, vol. 25, pp. 7046-7054, 2017.

[10] R. G. Heideman and P. V. Lambeck, "Remote opto-chemical sensing with extreme sensitivity: Design, fabrication and performance of a pigtailed integrated optical phase-modulated Mach-Zehnder interferometer system," Sens. Actuators B, vol. 61, pp. 100-127, 1999.

[11] K. Qin et al., "Slow light Mach-Zehnder interferometer as label-free biosensor with scalable sensitivity," Opt. Lett., vol. 41, pp. 753-756, 2016

[12] V. Toccafondo and C. J. Oton, "Robust and low-cost interrogation technique for integrated photonic biochemical sensors based on MachZehnder interferometers," Photon. Res., vol. 4, no. 2, pp. 57-60, 2016.

[13] S. Dante et al., "All-optical phase modulation for integrated interferometric biosensors," Opt. Express, vol. 20, pp. 7195-7205, 2012.

[14] Y. Marin et al., "Silicon-photonic interferometric biosensor using active phase demodulation," in Proc. SPIE, Front. Biol. Detection, Nanosens. Syst. X, 2018, vol. 10510, Paper 1051005

[15] A. Dandridge, A. B. Tveten, and T. G. Giallorenzi, "Homodyne demodulation scheme for fiber optic sensors using phase generated carrier," IEEE Trans. Microw. Theory Techn., vol. MTT-30, no. 10, pp. 1647-1653, Oct. 1982.

[16] N. Rouger, L. Chrostowski, and R. Vafaei, "Temperature effects on siliconon-insulator (SOI) racetrack resonators: A coupled analytic and 2-D finite difference approach," J. Lightw. Technol., vol. 28, no. 9, pp. 1380-1391, May 2010.

[17] J. E. Saunders et al., "Refractive indices of common solvents and solutions at 1550 nm," Appl. Opt., vol. 55, pp. 947-953, 2016.

Yisbel Eloisa Marin received the B.Sc. degree in electronic engineering from the Universidad Nacional Experimental Politecnica "Antonio Jose de Sucre," Barquisimeto, Venezuela, in 2010, and the M.Sc. degree in photonic networks engineering from both Aston University, Birmingham, U.K., and Scuola Superiore Sant'Anna, Pisa, Italy, in 2015. She is currently working toward the Ph.D. degree in emerging digital technologies, photonic technologies curriculum at the Institute of Information Communication and Perception Technologies, Scuola Superiore Sant' Anna. Her current research interests include optical fiber sensors and photonic-integrated circuits.

Veronica Toccafondo received the Graduate degree in physics from the University of Pisa, Pisa, Italy, in 2005, and the Ph.D. degree from the Scuola Superiore Sant'Anna, Pisa, Italy, in 2009. During this time, her research was focused on novel materials and pumping schemes for integrated waveguide amplifiers. She then spent 3 years as a Postdoctoral Research Fellow with the Nanophotonics Technology Center, Universidad Politecnica de Valencia, Valencia, Spain, working on integrated photonic biosensors. In 2013, she joined the Consorzio Interuniversitario per le Telecomunicazioni, Pisa, Italy, as a researcher, where she currently holds a permanent position. Her current research interests include novel advanced packaging techniques for integrated photonic devices, silicon integrated devices, as well as metrology.
Philippe Velha received the Graduate degree in engineering from the École Centrale de Lyon, Écully, France, in 2004, the M.Sc. degree in integrated digital electronics, and the Ph.D. degree in silicon photonics working for three different laboratories: Institut d'Optique, Palaiseau, France, Laboratoire des technologies de le Microélectronique (CNRS), Grenoble, France, and Silicium Nanoélectronique Photonique et Structures, Grenoble, France, from the CENG, in 2008. He is an established physicist whose expertise lies mainly in silicon photonics and nanofabrication. He became a Research Assistant with the University of Glasgow. In June 2013, he joined the newly created Silicon Photonics Group, Scuola Superiore Sant'Anna, Pisa, Italy, and became an Assistant Professor in 2017. He is a co-author of more than 70 scientific publications, 3 patents, and a book.

Simona Scarano received the M.Sc. degree in chemistry from the University of Pisa, Pisa, Italy, in 2002, and the Ph.D. degree from "Sensors and Biosensors" Laboratory, Via Giuseppe Moruzzi, 1, 56124 Pisa PI, Italy, in 2011. Then, she spent three years as a researcher with Gnosis S.p.a., Milan, Italy. Currently, she has a researcher position, working on affinity-based biosensors for clinical diagnostics, antidoping, and cultural heritage conservation. She coordinates the "Scientific Independence of Young Researchers" project funded by Italian Ministry for Education, University and Research, for the development of point-of-care nanosensors based on new synthetic bioreceptors coupled to localized surface plasmon resonance detection for clinical diagnostics.

Stefano Tirelli received the Graduate degree in physics from the University of Pisa, Pisa, Italy, and the Ph.D. degree in electrical engineering from the Swiss Federal Institute of Technology, Zürich, Switzerland, on the topic of gallium nitride transistors in 2014. His areas of expertise include nitride-based semiconductors, RF measurements, and nanofabrication technologies. Since 2015, he has been working as a Process Engineer with the InPhoTec Foundation, Pisa, Italy. His main activities are related to managing of plasma etching and deposition processes as well as process integration. His research interests include material science, device physics, and micro- and nano-fabrication technologies.

Alessandro Nottola received the Graduate degree in physics from the University "La Sapienza," Rome, Italy, in 1997. He then worked in public institutions as a researcher, in the fields of laser-driven plasma sources (University of Essex) and nanofabrication by electron-beam lithography (Italian National Research Council). From 2000 to 2009, he worked with Pirelli Labs, Milan, Italy, as a Lithography Process Engineer, for the development and prototyping of optical integrated circuits. From 2009 to 2012, he worked with Pramac Swiss SA, Riazzino, Switzerland, as the head of laser technology and a Front-End Process Engineer for the production of silicon thin film photovoltaic modules. From 2012 to 2015, he worked with RSE, Piacenza, Italy, in the Concentrated Photovoltaics and LED Laboratory, as an R\&D Engineer, developing frontend processes like lithography and lift-off for the postgrowth manufacturing of the concentrated thin-film III-V photovoltaic cells and modules. From 2015 to present, he has been working with the Inphotec facility, within the Scuola Superiore Sant'Anna, Pisa, Italy, as a Process and R\&D Engineer for the frontend process development of photonic integrated circuits. He currently manages the lithography processes for the development and prototyping of silicon-oninsulator and glass-on-silicon waveguides and components, and takes care of process integration, process transfer, and innovative processes. 
Yoonah Jeong received the Bachelor's degree in chemistry from Yonsei University, Seoul, South Korea, in 2011, and the M.A. degree in environmental management from Seoul National University, Seoul, South Korea, in 2013. She is working toward the Ph.D. degree at RWTH Aachen University, Aachen, Germany. Her research focuses on how to efficiently monitor the organic contaminants in the field of environmental chemistry. Alongside her dissertation research, she remains interested in state-of-the-art analytical techniques used for identification and quantification of a broad spectrum of chemicals.
Maria Minunni received the Degree in biology from Pisa University, Pisa, Italy, in 1988, and the Ph.D. degree in environmental science from the University of Florence, Florence, Italy, in 1994. From 1989 to 1999, she was a Visiting Scientist and a Post-Doc in several research laboratories in Europe; in particular, in Switzerland (1989-1990), with Nestlé Research Centre; in Sweden (1991-1992), with Pharmacia Biosensor AB, Uppsala, currently acquired by GE Healthcare; in Germany (1998-1999), with the Technical University of Munich, Institute of Life Sciences; and in Ireland (1995), with the University College UCC-Cork. In Italy, she was with the University of Florence (1995-1997) both in Chemistry and Pharmaceutical Science Departments. She is currently a Full Professor of analytical chemistry with the University of Florence, before being an Associate Professor (2011-2017) and a Researcher (2000-2011). She has authored 107 full papers with $H$ index 35 (source Scopus), and 18 book chapters. She is a Journal Board Member of Sensing and Bio-Sensing Research, Elsevier, and Frontiers in Chemistry. She is a referee and an evaluator for grant programs of several agencies including European and non-European; and a Referee for the main journals in analytical chemistry and sensors area.

Fabrizio Di Pasquale received the Degree in electronic engineering from the University of Bologna, Bologna, Italy, in 1989, and the Ph.D. degree in information technology from the University of Parma, Parma, Italy, in 1993.

From 1993 to 1998, he was with the Department of Electrical and Electronic Engineering, University College London, London, U.K., as a Research Fellow, working on optical amplifiers, wavelength division multiplexing (WDM) optical communication systems, and liquid crystal displays. After two years with Pirelli Cavi e Sistemi and two years with Cisco Photonics Italy, he moved to Scuola Superiore Sant'Anna, Pisa, Italy, where he is currently a Full Professor of telecommunications. He is the Co-Founder and the President of INFIBRA TECHNOLOGIES, a spin-off company of Scuola Superiore Sant'Anna, developing and marketing advanced fiber optic sensor systems. He has filed 20 international patents and he is the author and co-author of more than $200 \mathrm{sci}-$ entific journals and conference papers. His current research interests include optical fiber sensors, silicon photonics, optical amplifiers, WDM transmission systems, and networks. He is on the Board of Reviewers for the IEEE PHOTONICS TECHNOLOGY LETTERS, the IEEE/OSA JOURNAL OF LIGHTWAVE TECHNOLOGY, the IEEE SENSORS JOURNAL, Optics Communications, Optics Express, and Optics Letters.

Claudio J. Oton received the Ph.D. degree from the University of La Laguna, San Cristóbal de La Laguna, Spain, in 2005. Then, he spent 4 years with the Optoelectronics Research Centre, Southampton, U.K., where he worked on integrated optical amplifiers and lasers on silicon as a Marie Curie Post-Doctoral Fellow. In 2009, he joined the Nanophotonics Technology Centre, Universidad Politecnica de Valencia, Valencia, Spain, where he studied nonlinear silicon photonic devices. Finally, since 2012, he has been an Assistant Professor with TeCIP Institute, Scuola Superiore Sant'Anna, Pisa, Italy. He is the author of more than 120 scientific papers and conference contributions. His current research interests include optical fiber sensors and silicon photonic integrated devices. 